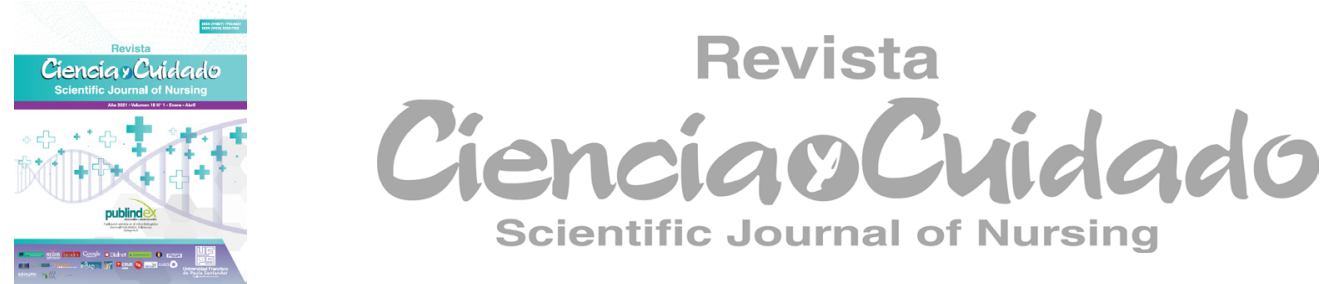

Articulo de Reflexión

\title{
Enfermería, pandemia y fatiga por compasión: una reflex- ión general sobre el 2020
}

\author{
Nursing, Pandemic, and Compassion Fatigue: A General Reflection on 2020 \\ Enfermagem, pandemia e fadiga por compaixão: uma reflexão geral sobre 2020
}

Diana Isabel Cáceres-Rivera

\section{Resumen}

La actual pandemia por COVID-19 ha significado para los profesionales de la enfermería un escenario en el que se ha hecho evidente el impacto que el ejercicio profesional puede tener sobre su calidad de vida. Justo cuando el 2020 fue escogido por la Organización Mundial de Salud como el año de la enfermería, se ha evidenciado un panorama poco favorecedor para el ejercicio del cuidado, en el que no sólo se han visto afectados pacientes, familias, sistemas de salud, sino también los propios profesionales que prestan atención a pacientes diagnosticados con el nuevo virus. En el presente artículo de reflexión se realizó un análisis de uno de los efectos de las condiciones actuales de trabajo de los profesionales de enfermería: la fatiga por compasión, la cual constituye una afectación de la calidad de vida; también se presentan las recomendaciones actuales disponibles, para hacer frente a este fenómeno en Colombia.

Palabras clave: Enfermeras clínicas, atención de enfermería, estrés laboral, agotamiento profesional, satisfacción en el trabajo.

\footnotetext{
Abstract

The current COVID-19 pandemic has meant for nursing professionals a scenario in which the impact that professional practice can have on their quality of life has become evident. Just when 2020 was chosen by the WHO (World Health Organization) as the year of nursing, an unflattering panorama has been evidenced for the exercise of care in which not only patients, families, health systems have been affected, but also the professionals who care for patients diagnosed with the new virus. In this reflection article, an analysis of one of the effects of the current working conditions of nursing professionals was carried out: compassion fatigue, which constitutes
}

Autor de correspondencia*

$\mathbf{1}^{*}$ Enfermera. Magister en Enfermería, Doctora en Biomedicina. Profesora Investigadora. Universidad Cooperativa de Colombia. Bucaramanga. Correo: dianai.caceres@ucc.edu.co (iD)

Recibido: 1 septiembre 2020

Aprobado: 4 noviembre 2020

Para citar este articulo:

Cáceres-Rivera DI. Enfermería, pandemia y fatiga por compasión: una reflexión general sobre el 2020. Rev. cienc. cuidad. 2021;18(1):116-123. https://doi.org/10.22463/17949831.2674

(C) Esta obra está bajo licencia internacional CCBY-NC-ND

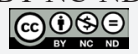


an impairment of the quality of life, as well as the current recommendations available to make faced with this phenomenon in Colombia.

Keywords: Nurse Clinicians, Nursing Care, Occupational Stress, Burnout Professional, Job Satisfaction

\begin{abstract}
Resumo
A atual pandemia pela COVID-19 tem significado para os enfermeiros profissionais um senário que evidenciou o impacto do exercício profissional pode ter sobre a qualidade de vida. Justo quando o 2020 foi escolhido pela Organização Mundial da Saúde como o ano da enfermagem, tem se evidenciado um panorama pouco favorecedor para o exercício de prestar cuidados, onde não só os pacientes, famílias e sistemas de saúde têm sido os afetados, como também os profissionais que prestam atenção aos diagnosticados com o novo vírus. No presente artigo de reflexão realizou-se uma análise dos efeitos das condições atuais de trabalho dos profissionais de enfermagem: a fadiga por compaixão, constituindo uma afetação da qualidade de vida; também se apresentam as recomendações atuais disponíveis, para fazer frente a esse fenômeno em Colômbia.
\end{abstract}

Pavras-chave: Enfermeiras clínicas, cuidados de enfermagem, estresse ocupacional, esgotamento profissional, satisfação no emprego.

\section{Introducción}

Los profesionales de la enfermería son susceptibles de presentar cambios en su calidad de vida, específicamente fatiga por compasión y síndrome de burnout, entre otras condiciones. Estas alteraciones se derivan de las situaciones que se generan en la práctica diaria como lo es la generación de sentimientos de empatía, lo que conlleva a interiorizar y en algunas ocasiones a apropiarse de los problemas de las personas, sus familias y la comunidad de quienes se les brinda cuidado. Adicionalmente, valores como la compasión y la empatía son fomentados durante la formación en pregrado como características deseables en el ejercicio profesional, lo que los lleva a ser más vulnerables a la exposición de riesgos emocionales, producto del ejercicio disciplinar (1).

En un contexto como el colombiano, con los retos que representa el cuidado en los diferentes niveles de atención, el profesional de enfermería está expuesto a situaciones como el sufrimiento, el dolor, la angustia y la muerte; además de las intensas demandas físicas y emocionales propias del trabajo con seres humanos. Lo anterior genera una carga emocional a la que pocas profesiones se ven enfrentadas (2).

Actualmente, cuando los ojos del mundo están puestos sobre la primera línea de batalla contra el COVID-19, la cual involucra necesariamente a la enfermería como la fuerza laboral con mayor número de profesionales enfrentando la pandemia, es importante revisar cuáles son los efectos que tiene este tipo de situaciones en los y las enfermeras y qué se podría hacer en nuestro medio.

Durante este 2020 han sido frecuentes los reportes de noticias en donde se muestran agresiones físicas y verbales al personal de salud que atiende a pacientes con COVID-19. Esta situación ha sido recurrente en general en Latinoamérica y Colombia lastimosamente, no ha sido la excepción (3). No siendo suficiente la exposición a estos daños físicos y psicológicos, es muy bien conocida la crisis del sector salud en medio de la cual trabajan muchos de los profesionales de la enfermería, donde en algunos escenarios se labora sin recibir una remuneración digna e incluso bajo amenazas y acoso de tipo laboral, en un mundo en donde cada vez los recursos para la salud son menos, forzándolos a condiciones laborales precarias, con recortes en el personal y aumento de la razón enfermera-paciente (4).

Este panorama parece desolador y como es de esperarse ha repercutido en la salud de los profesionales de la enfermería. Esto se ha evidenciado en diferentes investigaciones en donde se ha evaluado el nivel de fatiga por compasión e incluso se han identificado el síndrome de Burnout y estrés pos traumático. El presente artículo 
se elaboró con el objetivo de realizar una reflexión en torno al impacto de situaciones como una pandemia y lo que se podría hacer para cuidar a nuestros propios colegas. Igualmente se quiere contribuir en hacer evidentes nuestras necesidades y oportunidades, a fin de fortalecer la disciplina en nuestro país.

\section{Desarrollo}

\section{Historia de la fatiga por compasión}

En 1971 Figley estudió por primera vez los efectos en la salud de las enfermeras que atendieron veteranos de la guerra de Vietnam en las décadas de los 60 y 70, encontrando sentimientos de culpa y remordimiento, lo que más adelante se traduciría como síndrome de estrés pos traumático, derivado de la exposición continuada a las historias de dolor y sufrimiento de los pacientes (5). Este escenario es semejante a la situación actual en relación con la pandemia, en donde una nueva guerra se está librando en contra de un nuevo tipo de enfermedad y todos los desafíos a lo que esto conlleva. Así, mientras una nueva vacuna se logre desarrollar, probar e implementar, esta batalla podría durar algunos meses más, e incluso años, teniendo como protagonistas a los profesionales de la salud, por tanto es necesario un replanteamiento de las actuales condiciones laborales.

A este síndrome descrito por Figley más adelante se le conoció como un estado de agotamiento y disfunción biológica, psicológica y social, resultado de la exposición prolongada al estrés y todo lo que ello evoca (6). Posteriormente se identificaron una serie de síntomas que se manifestaban en reacciones cognitivas, emocionales y comportamentales que algunos autores han clasificado en tres grupos: psicológicos, cognitivos e interpersonales (7). Todas estas condiciones fueron unificadas bajo el concepto de calidad de vida de los profesionales de la salud y en particular en la satisfacción o fatiga que puede tener un profesional de la salud secundario a su ejercicio profesional. Algunos de estos síntomas son evidentes e incluso exacerbados en los profesionales de enfermería en diversos contextos y situaciones. Actualmente se ha empezado a identificar en aquellos profesionales que se encuentran en las unidades para atención de pacientes con COVID-19 como lo describen Pouralizadeh et al., quienes encontraron altos niveles de ansiedad y depresión en profesionales de enfermería en 25 hospitales de medio oriente (8).
El término de fatiga por compasión fue utilizado por primera vez en una revista de enfermería en 1992 para describir situaciones en las que las enfermeras presentaban dificultad para experimentar sus propios sentimientos o sentían impotencia y rabia en respuesta a su actividad diaria en un servicio de emergencias en donde les suponía un estrés constante (9). Desde entonces, diversos autores han descrito esta situación como característica del personal de enfermería en diferentes ámbitos laborales o situaciones de cuidado (10).

Se han desarrollado diversos instrumentos de medición de los efectos negativos de las situaciones estresantes en el trabajo, siendo muy reconocida la Escala de Burnout de Maslach (11). Sin embargo, este concepto ha evolucionado a un fenómeno mayor que incluye términos como "calidad de vida del profesional de salud" desarrollado por Stamm en 2010, con el que se logra identificar no sólo la fatiga por compasión, el síndrome de Burnout y el trauma secundario, sino también la satisfacción por compasión, introduciendo un concepto positivo a las experiencias de cuidado de los profesionales de salud (12). Este último concepto hace referencia a un estado en el cual el profesional de salud encuentra bienestar personal y profesional en el ejercicio de sus actividades diarias como cuidador.

En estos momentos, diagnosticar la "satisfacción por compasión" parece algo imposible de conseguir, pero es allí donde el gremio debe implementar medidas locales y globales realizando movimientos como el "Nursing Now," con verdaderas causas que conlleven a resultados que permanezcan en el tiempo. Dicha definición, aunque suene utópica pudiera concretarse con la implementación de estrategias que se han experimentado en instituciones en donde la salud del trabajador ha sido considerada como vital para brindar una atención de calidad.

\section{Factores protectores y de riesgo}

La relación entre el ambiente de trabajo y su incidencia en la aparición de condiciones como la fatiga por compasión, no es del todo clara a pesar de que se han descrito las repercusiones que pueden tener en la salud física y mental de los profesionales de salud. Algunos estudios sugieren que las condiciones del trabajo pudieran dar origen a sentimientos tanto positivos como negativos. Por ejemplo, se ha descrito que el desarrollo de un adecuado liderazgo, así como los reconocimientos y agradecimientos en el entorno laboral pudieran ser un factor protector (13). Un programa de incentivos 
profesionales, académicos e incluso económicos también sería una adecuada herramienta para proteger a los profesionales de enfermería de los efectos de la fatiga, relacionada con la actual situación. Sin embargo y hasta el momento en el que se redactó este artículo, iniciativas como los bonos propuestos por el Gobierno Nacional al personal de salud enfrentando al COVID-19, aún no se habían materializado completamente.

En relación con este aspecto económico, y aunque no es el único factor protector, se sabe que se convierte en uno de los más influyentes para prevenir la fatiga por compasión, ya que en nuestro contexto, en la mayoría de hogares se depende del ingreso de dos o más miembros de la familia para mantener un hogar, por lo que un buen salario es determinante a la hora de identificarse y sentirse a gusto con el trabajo. Se ha descrito que una baja remuneración tiene un impacto negativo sobre el rendimiento laboral y la salud mental de profesionales de la enfermería (14). En este sentido, la Organización Colegial de Enfermería (OCE) en su último informe encontró un salario promedio de $\$ 2.474 .002$ en general, para los profesionales de enfermería (15). Esta remuneración dista mucho de la recibida en otros países que se encuentran en el ranking de los 15 con mejor remuneración para las enfermeras como se muestra en la Tabla 1, en donde solo un país de Latinoamérica logra entrar en esta limitada lista.

Tabla 1. Países con mejor remuneración para los profesionales de enfermería.

\begin{tabular}{cc}
\hline País & Salario bruto en USD por año \\
\hline Luxemburgo & $\$ 80.000$ \\
USA & $\$ 70.000$ \\
Irlanda & $\$ 64.000$ \\
Australia & $\$ 56.000$ \\
Dinamarca & $\$ 54.000$ \\
Noruega & $\$ 52.000$ \\
Canadá & $\$ 51.000$ \\
Bélgica & $\$ 50.000$ \\
Nueva Zelanda & $\$ 49.000$ \\
Chile & $\$ 47.000$ \\
Alemania & $\$ 47.000$ \\
España & $\$ 45.000$ \\
Holanda & $\$ 46.000$ \\
Israel & $\$ 45.000$ \\
Reino Unido & $\$ 44,000$ \\
\hline
\end{tabular}

Fuente: La autora. Datos tomados de OCDE Health Stadistics 2019. 
Existen condiciones que disminuyen el riesgo de presentar fatiga por compasión como la edad, un nivel de formación pos gradual mayor, tener varios años de experiencia y jornadas laborales cortas, diurnas y con contratos a término indefinido (16). Adicionalmente, algunas estrategias como los programas de apoyo institucional, oportunidades educativas y soporte profesional y psicológico, pueden contribuir a la prevención de la fatiga por compasión en ambientes estresantes (17). En relación con este tema ha sido interesante ver cómo el nivel de formación de las enfermeras colombianas ha incrementado, aumentado considerablemente el número de magísteres y doctoras en el país (18). Sin embargo, este esfuerzo por adquirir conocimientos con el ánimo de innovar en el cuidado, surge en su mayoría de una motivación personal o familiar en la que la financiación depende de la economía personal.

Una organización gremial, como la colombiana que poco a poco se ha hecho más sólida, podría consolidar estrategias de educación si se contara con un mayor apoyo por parte de entidades estatales y privadas. En este punto se debe demostrar que, a mayor formación, habrá mejores indicadores de salud y sobretodo, un excelente cuidado. En un entorno en donde la salud se revisa en cifras, demostrar que invertir en el personal de enfermería trae beneficios podría ser una estrategia para obtener mayores recursos.

Por otro lado, como factores de riesgo se han identificado aspectos tales como el entorno del paciente, el del cuidador, el laboral y algunos antecedentes personales. En relación a esto y retomando el concepto de satisfacción por compasión, Herrera-Amaya describió que el ámbito hospitalario goza de mayor satisfacción por el trabajo en comparación con el comunitario (19). Esto podría deberse a las múltiples dificultades de aquellos profesionales dedicados a la salud pública, lo que conlleva a sentimientos de frustración en un trabajo en el que se necesitan muchos años, en la mayoría de los casos, para ver resultados.

En cuanto al género de los profesionales se ha descrito que existe un mayor riesgo para las mujeres, sobre todo para aquellas que tienen contacto con pacientes críticos, pacientes pediátricos, unidades de cuidado paliativo, profesionales con antecedentes previos de situaciones estresantes y para quienes desconocen la existencia de la fatiga por compasión, el Burn out o similares y trabajan en situaciones con limitación de recursos (2023). Este escenario plantea una situación crítica para las mujeres que en enfermería son las más numerosas en los servicios mencionados y quienes además según la OCE reciben un salario hasta un 15\% inferior al de los hombres.

En Colombia, que ha sido reconocido como líder en salud en Latinoamérica, se encuentran los dos extremos: instituciones con un alto nivel de tecnología con recursos disponibles casi ilimitados e instituciones con lo mínimo para atender situaciones de un primer nivel de complejidad. Desde este punto de vista se debe revisar, no sólo el entorno sino todos los factores que influyen como la razón enfermera-paciente y estrategias de salud ocupacional entre otras. Además, hay que efectuar un adecuado proceso de selección, inducción y re inducción que podrían identificar y tratar factores de riesgo a tiempo, constituyéndose en una responsabilidad de las instituciones empleadoras.

Finalmente, es necesario resaltar la importancia de otro factor que no ha sido descrito a profundidad y está relacionado con la aparición de la fatiga por compasión, tiene que ver con la empatía que puede generarse en la relación enfermera paciente. Como lo describe Hudnall en la explicación del cuestionario para medir este fenómeno, él como el profesional se relaciona y responde a esta interacción con sus sujetos de cuidado, lo que será determinante en la aparición de satisfacción o fatiga. Referente a este aspecto, en una revista especializada en enfermería, se describe la importancia de la empatía, al afirmar que los profesionales de la salud se deben interesar por lo que el paciente está pensando o sintiendo, ya que son ellos quienes están llamados a responder con sensibilidad al sufrimiento del otro y a actuar enfocándose, particularmente, en el alivio y el beneficio del paciente (24). Es necesario pensar también en el impacto del sufrimiento de otros sobre el propio profesional de la enfermería y en los sentimientos que se generan. En este sentido una de las situaciones que llevan a la fatiga por compasión consiste en el no poder separar la vida profesional de la personal.

\section{Intervenciones efectivas para la fatiga y qué tan le- jos estamos en Colombia}

Se desconocen muchos aspectos sobre la situación laboral de los profesionales de la enfermería y la repercusión sobre su salud mental y física. Sin embargo, desde hace unos años se han venido identificando necesidades de los profesionales de la enfermería, a través de estudios que han evaluado entre otros aspectos, la calidad de vida, la presencia del síndrome de Burnout y la carga 
laboral. En ellos, se evidencia una alta carga de trabajo, necesidad de mejorar los espacios laborales y un mayor riesgo en profesionales que trabajan en áreas como urgencias y unidades de cuidado intensivo (25-28).

Acerca de qué se está haciendo por la enfermería, con el movimiento Nursing Now, se busca reivindicar la disciplina haciéndola más visible. Curiosamente y como una casualidad paradójica, la OMS declaró el 2020 como el año de la enfermería. Año en el que el COVID-19 se ha encargado de hacer visible los rostros de enfermeras $\mathrm{y}$ enfermeros en todo el mundo; algunos con reconocimientos y otros lastimosamente, en homenajes póstumos. Esto debe suscitar preguntas acerca de lo que se requiere y cómo se debe materializar en acciones que mejoren la calidad de vida de los profesionales de la enfermería.

Al respecto se han efectuado estudios en donde se han evaluado intervenciones que buscan mitigar o prevenir la fatiga por compasión, tales como la musicoterapia, la estimulación transcraneana de corriente continua y la Transcranial Direct Current Stimulation (tDCS), sin mucho éxito. Sin embargo, se ha experimentado con otras más eficaces para disminuir la fatiga por compasión como el yoga, mindfullness, meditación guiada y terapias de resiliencia, las cuales deben tener en promedio una duración de 4 semanas $(29,30)$. En este sentido, a la luz de las actuales circunstancias y en el actual contexto, este tipo de intervenciones podría no ajustarse a las necesidades del país en términos de disponibilidad de recursos y tiempo, ya que como se conoce, los turnos asistenciales oscilan entre las 8 y 12 horas, requiriendo muchas veces horas extras no remuneradas ni recompensadas.

Estos hallazgos a la luz de la realidad colombiana tampoco podrían ajustarse ya que los escenarios son totalmente diferentes. Los estudios en mención se han desarrollado en países como Reino Unido y otros países de Europa donde la razón enfermera-paciente es en su mayoría uno a uno y la disponibilidad de recursos, las condiciones y la remuneración laboral es mucho mejor. Al respecto, después del análisis de los factores de riesgo y los protectores, las medidas más urgentes serían respectivamente aquellas que conduzcan a una mejor condición laboral y una mejor remuneración, acordes al nivel de formación profesional. También, hay que garantizar una formación continua y un verdadero programa de riesgos laborales, en donde no solamente se valore el riesgo físico derivado de la atención en salud, sino también los riesgos y alteraciones en la calidad de vida.
Por otro lado, y haciendo una reflexión más profunda de cómo se podrían promover factores protectores, es aconsejable que este sea un tema a tener muy en cuenta en la formación de los profesionales en enfermería. Como se describió anteriormente, conocer la existencia de las alteraciones derivadas del ejercicio profesional pueden convertirse en un factor protector, por lo que el propio cuidado de la salud como trabajadores de la salud debería ser un tema que se discuta en las aulas de clase, lo que conllevaría a reforzar el liderazgo laboral, no solo el que se espera como buenos trabajadores, sino como parte de un gremio que requiere de una mayor unidad.

\section{Conclusiones}

- Es prioritario evaluar y garantizar condiciones laborales óptimas para los profesionales de la enfermería, no sólo para mitigar los efectos de la actual contingencia, sino también en preparación para futuros escenarios para los cuales aún no se está preparado. Diagnosticar e intervenir alteraciones como la fatiga por compasión se hace necesario con el ánimo de mejorar la calidad de vida.

- Se deben pensar intervenciones enfocadas en la recuperación social, económica y mental de las enfermeras asistenciales, posterior a la fase crítica de la pandemia, a través del liderazgo en los diferentes escenarios políticos y sociales de nuestro país, con el fin de lograr una verdadera transformación de las condiciones laborales.

- Es necesario conocer las propias necesidades de cuidado de los profesionales de la enfermería, así como la eficacia de todas aquellas iniciativas que mejoren su calidad de vida.

- Una limitación de la presente reflexión tiene que ver con la literatura disponible en Colombia sobre las condiciones laborales de los profesionales de la enfermería y su impacto, ya que la mayoría de los artículos son muy antiguos, por lo que se recomienda continuar con la investigación sobre este tema.

\section{Conflicto de intereses}

La autora declara no tener ningún conflicto de interés 


\section{Referencias Bibliográficas}

1. Gómez-Urquiza JL. Prevalencia e identificación de factores de riesgo de síndrome de Burnout en profesionales de Enfermería en servicios de Oncología. [Tesis Doctoral]. Granada: Universidad de Granada; 2017.

2. Infante-Ochoa I, Moret-Montano A, Curbelo-Heredia I. Satisfacción del personal de enfermería en servicios de oncología. Rev. Cubana Salud Pública 2007; 33 (3). https://doi.org/10.1590/S0864-34662007000300011

3. Bedoya JCM. COVID-19: the pandemic of abuse against health personnel in times of pandemic. IJEPH. 2020; 3(1): e-6276. https://doi.org/10.18041/2665-427X/ ijeph.1.6276

4. Campos-Vidal JF, Cuartero-Castañer ME, Riera-Androver JA, Cardona-Cardona J. Cuidado profesional y mecanismos paliativos del desgaste por empatía. Universidad de las Islas Baleares [Internet], España. 2016.

5. Figley CR. Treating compassion fatigue. New York. Brunner-Routledge 2002.

6. Lynch SH, Lobo LM. Compassion fatigue in family caregivers: a Wilsonian concept analysis. Journal of Advanced Nursing 2012; 68(9): 2125-2134. https://doi.org/10.1111/j.1365-2648.2012.05985.X

7. Gómez-Martínez S, Gil-Juliá B, Castro-Calvo J, Ruiz-Palomino E, Ballester-Arnal R. "Fatiga compasiva en el personal de enfermería de una unidad de cuidados intensivos." [Tesis de Maestría]. Valencia: Universidad Jaume I; 2015.

8. Pouralizadeh M, Bostani Z; Maroufizadeh S; Ghanbari A; Khoshbakht M; Alavi SA; et al. Anxiety and Depression and the Related Factors in Nurses of Guilan University of Medical Sciences Hospitals During COVID-19: A Web-based Cross-Sectional Study. Int J Afr Nurs Sci. 2020; 11(13):100-233. https://doi.org/10.1016/j.ijans.2020.100233

9. Joinson, C. Coping with compassion fatigue. Nursing, 1991; 22: 116-122.

10. Fernández, CM. "Fatiga por compasión entre el personal de enfermería en los cuidados al final de la vida." N. Punto 2019: 2(21):49-69

11. Méndez I, Secanilla E, Martínez JP, Navarro J. Estudio comparativo de burnout en cuidadores profesionales de personas mayores institucionalizadas con demencias y otras enfermedades. European Journal of Investigation in Health, Psychology and Education 2011; 1(2): 61-70. https://doi.org/10.30552/ejihpe.v1i2.17

12. Stamm, BH. "The concise ProQOL manual." (2010): 1-30.

13. Lesly K, Todd M. Compassion fatigue and the healthy work environment. ACN Adv Crit Care (2017) 28 (4): 351-358. https://doi.org/10.4037/aacnacc2017283

14. Acosta-Fernández M, Parra-Osorio L, Restrepo-García JI, Pozos-Radillo BE, de los Ángeles Aguilera-Velasco M, Torres-López TM. Condiciones psicosociales, violencia y salud mental en docentes de medicina y enfermería. Salud Uninorte. 2017; 33(3): 344-354.

15. Organización Colegial de Enfermería. ¿Cuál es la situación salarial de las enfermeras en Colombia?. OCE. Boletin-02. https://www.oceinfo.org.co/organizacion-colegial-de-enfermeria/boletin-oce/214-cual-es-la-situacion-salarial-de-las-enfermeras-en-colombia

16. Harris C, Griffin MT. Quinn Nursing on Empty, Journal of Christian Nursing. 2015; 32(2): 80-87. https://doi. org/10.1097/CNJ.0000000000000155

17. Zhang YY, Zhang C, Han XR, Li W, Wang YL. Determinants of compassion satisfaction, compassion fatigue and burn out in nursing: A correlative meta-analysis. Medicine 97.26 (2018). https://doi.org/10.1097/ MD.0000000000011086

18. Ministerio de Educación. Sistema Nacional de Información de la Educación Superior. Informe SNIES 2018 [Internet] [citado 2 de septiembre de 2020]. Disponible en: https://snies.mineducacion.gov.co/portal/ 
19. Herrera-Amaya G, Manrique-Abril F. Satisfacción laboral de enfermería. Diferencias de ámbitos en atención primaria en salud y cuidado intensivo. Duazary 16.2 (2019): 74-86. https://doi.org/10.21676/2389783X.2943

20. Kim MR, Seomun G. "Relationships among burnout, job satisfaction, organizational commitment and turnover intention to resign in hospital nurses. J Korean Acad Nurs 2013; 22(2): 93-101. https://doi.org/10.5807/ kjohn.2013.22.2.93

21. Komachi MH, Kamibeppu K, Nishi D, Matsuoka Y. Secondary traumatic stress and associated factors among Japanese nurses working in hospitals. Int J Nurs Pract 2012; 18(2): 155-63. https://doi.org/10.1111/j.1440172X.2012.02014.X

22. Meyer-Rica ML, Li A, Klaristenfeld J, Gold JI. Pediatric novice nurses: examining compassion fatigue as a mediator between stress exposure and compassion satisfaction, burnout, and job satisfaction. J Pediatr Nurs 2014. https://doi.org/10.1016/j.pedn.2013.12.008

23. Al-Barmawi MA, Subih M, Salameh O, Sayyah Yousef SN, Shoqirat N, Abdel-Azeez Eid Abu Jebbeh R. Coping strategies as moderating factors to compassion fatigue among critical care nurses. Brain Behav. 2019;9(4): e01264. https://doi.org/10.1002/brb3.1264

24. Triana-Restrepo M. La empatía en la relación enfermera-paciente. Avances en Enfermería 35.2 (2017): 121122. http://dx.doi.org/10.15446/av.enferm.v35n2.66941

25. Muñoz AI, Velásquez MS. Síndrome de quemarse por el trabajo en profesionales de enfermería, Bogotá, Colombia. Rev. Fac. Nac. Salud Pública [Internet]. 21 de mayo de 2016 [citado 2 de septiembre de 2020]; 34(2):202-11. Disponible en: https://revistas.udea.edu.co/index.php/fnsp/article/view/21798

26. Suescún-Carrero S, Sarmiento G, Alvarez L, Lugo M. Calidad de vida laboral en trabajadores de una Empresa Social del Estado de Tunja, Colombia. Rev. Méd. Risaralda 2016; 22 (1):14-17

27. Matabajoy-Montilla JM, Matabachoy-Tulcán SM, Obando-Guerrero LM. Procesos de desarrollo del talento humano en una clínica de especialidades de Pasto, Colombia. Universidad y Salud 2018; 20(1): 26-36. http:// dx.doi.org/10.22267/rus.182001.106.

28. Cáceres D, Torres C, Cristancho L, López L. Carga laboral de los profesionales de enfermería en unidad de cuidados intensivos. Estudio descriptivo: «CARETIME». Acta Colomb Cuid Intensivo. 2020; 20(2): 92-7. https://doi.org/10.1016/j.acci.2019.12.002

29. Cocker F, Joss N. Compassion Fatigue among Healthcare, Emergency and Community Service Workers: A Systematic Review. Int J Environ Res Public Health. 2016 Jun 22;13(6):618. https://doi.org/10.3390/ijerph13060618

30. Delaney MC. Caring for the caregivers: Evaluation of the effect of an eight-week pilot mindful self-compassion (MSC) training program on nurses' compassion fatigue and resilience. PLoS One. 2018; 13(11):e0207261. https://doi.org/10.1371/journal.pone.0207261 